\title{
Padrones de Inervación y Localización Biométrica de los Puntos Motores del Músculo Bíceps Braquial en el Hombre
}

\author{
Innervation Patterns and Biometric Localization of the \\ Motor Points of the Brachial Biceps Muscle in Man
}

"Olave, E.; ${ }^{* *}$ Gabrielli, C. \& ${ }^{* *}$ Braga, M. T. T.

OLAVE, E.; GABRIELLI, C. \& BRAGA, M. T. T. Padrones de inervación y localización biométrica de los puntos motores del músculo bíceps braquial en el hombre. Int. J. Morphol., 27(2):495-501, 2009.

RESUMEN: La inervación del músculo bíceps braquial se torna importante debido a su constitución y a la acción que ejerce a nivel del codo, sobretodo cuando existen lesiones en nervios espinales o médula espinal alta. Con el objetivo de conocer con detalles la disposición de sus nervios y localizar desde el punto de vista biométrico sus puntos motores, se estudiaron 46 miembros superiores de 23 cadáveres formolizados de individuos brasileños, adultos, en los cuales se consideró el número de ramos, longitud, nivel de origen y puntos de penetración en el vientre muscular respecto a una línea trazada entre los epicóndilos humerales (LBE). El nervio musculocutáneo envia un ramo para el músculo en estudio como un ramo común que luego se dividió para sus dos cabezas en 19 casos (41,3\%); ramos independientes para cada cabeza se encontraron en las muestras restantes $(58,7 \%)$. Con respecto al número de ramos, la cabeza larga recibió un ramo (R1) en 35 casos (76,1\%), dos ramos (R1 y R2)en 9 casos $(19,6 \%)$ y tres (R1,R2 y R3) en 2 casos $(4,3 \%)$; para la cabeza corta se observó un ramo en 39 casos $(84,7 \%)$, dos en 5 casos (10,9\%) y tres en 2 casos $(4,3 \%)$. Cada uno de los ramos dirigidos a las cabezas del músculo bíceps se dividió en ramos secundarios (RS). Para la cabeza corta, el ramo principal se dividió en 2 RS en 20 casos; en 3 RS en 13 casos; en 4 RS en 4 casos, siendo directo sin dividirse en 2 casos. Para la cabeza larga el ramo principal se dividió en 2 RS en 16 casos; en 3 RS en 13; en 4 RS en 3; en 5 RS en un caso y en 6 RS en 1 caso y en una muestra, el ramo principal penetró en el vientre muscular sin dividirse. Los RS penetraron a niveles variables en el vientre muscular. Los resultados obtenidos pueden servir de referencia para procedimientos de punción en los puntos motores o en estudios electromiográficos.

PALABRAS CLAVE: Músculo bíceos braquial; Biometría; Puntos motores.

\section{INTRODUCCIÓN}

El conocimiento de la inervación motora de los músculos esqueléticos es de suma importancia cuando se producen lesiones en nervios espinales, cerebro o medula espinal alta. En el caso de la flexión del codo, si hay compromiso en esta función, la localización de los ramos del nervio musculocutáneo y sus puntos motores se torna importante, debido a que en las flexiones espásticas del codo se pueden administrar anestésicos locales y agentes neurolíticos en los puntos motores de los $\mathrm{mm}$. bíceps braquial y braquial para disminuir tal acción (Buchanan \& Erikson, 1996).

Descripciones generales sobre los ramos musculares mencionados han sido dadas por Testut \& Latarjet (1969); Williams et al. (1995); Moore \& Dalley (2002), habiéndose publicado trabajos más específicos que han aportado mayores detalles sobre estos ramos, principalmente sobre aquellos que inervan a las dos cabezas del músculo bíceps braquial (Yang et al.,1995; Buchanan \& Erikson; Chiarapattanakon et al., 1998; Pacha Vicente et al., 2005; Kwolczak-McGrath et al., 2008) quienes han señalado que existe un ramo común para ambas cabezas del $\mathrm{m}$. bíceps braquial en un porcentaje alto de individuos y ramos independientes para cada cabeza en un porcentaje menor.

Con el propósito de complementar el conocimiento anatómico y biométrico de los ramos mencionados, se realizó el estudio de éstos, considerando su número, longitud, nivel de origen y puntos de penetración el vientre muscular.

\footnotetext{
* Facultad de Medicina, Universidad de La Frontera, Chile.

** Departamento de Morfología, Universidad Federal de Santa Catarina, Brasil.
} 


\section{MATERIAL Y MÉTODO}

Se utilizaron 46 miembros superiores de 23 cadáveres formolizados de individuos adultos, de ambos sexos (21 masculino; 2 femenino). Primeramente, se registró la distancia entre la parte más prominente del acromion y una línea horizontal trazada entre partes más prominentes de los epicóndilos humerales (línea biepicondilar, LBE), para luego realizar la disección de la axila y el brazo, identificando el trayecto y los ramos que emite el nervio musculocutáneo para el músculo bíceps braquial. Cuando identificados, se registró el número de ramos para cada cabeza y las distancias entre los puntos de origen y penetración en el vientre muscular de los ramos mencionados en relación a la LBE. Para la localización biométrica de los puntos motores, la distancia acromion-LBE se dividió en tercios proximal, medio y distal, los cuales se dividieron una vez más en tres partes, para localizar con más precisión a los puntos mencionados. Los datos obtenidos fueron tratados estadísticamente comparando los resultados entre lados.

\section{RESULTADOS}

Los nervios dirigidos al músculo bíceps braquial proceden del nervio musculocutáneo y se presentó como un ramo común que luego se dividió para sus dos cabezas en 19 casos (41,3\%), 8 en el lado derecho y 11 en el izquierdo (Fig. $1)$; ramos independientes para cada cabeza se encontraron en las muestras restantes (58,7 \%), 15 en el lado derecho y 12 en el izquierdo (Fig. 2).

Con respecto al número de ramos, la cabeza larga recibió un ramo (R1) en 35 casos $(76,1 \%)$, dos ramos (R1 y R2)en 9 casos (19,6\%) y tres (R1,R2 y R3) en 2 casos $(4,3 \%)$; para la cabeza corta se observó un ramo en 39 casos $(84,7 \%)$, dos en 5 casos $(10,9 \%)$ y tres en 2 casos $(4,3 \%)$.

La longitud promedio del ramo dirigido a la cabeza larga (en los casos con un ramo) fue de $16,5 \mathrm{~mm}$ (DS 8,7) en el lado derecho y de 15,1 mm (DS 9,3) en el izquierdo; para la cabeza corta fue de $11,7 \mathrm{~mm}$ (DS 6,2) en el lado derecho y de 12,7 mm (DS 6,8) en el izquierdo. Al comparar los lados, no hubo diferencias estadísticamente significativas.

Las distancias entre el origen del ramo muscular y la LBE se muestran en la Tabla I.

Cada uno de los ramos dirigidos a las cabezas del músculo bíceps se dividió en ramos secundarios (RS, Fig. 3). Para la cabeza corta, el ramo principal se dividió en 2 RS en 20 casos; en 3 RS en 13 casos; en 4 RS en 4 casos, siendo directo sin dividirse en 2 casos.

Para la cabeza larga el ramo principal se dividió en 2 RS en 16 casos; en 3 RS en 13; en 4 RS en 3; en 5 RS en un caso y en $6 \mathrm{RS}$ en 1 caso. En una muestra, el ramo principal penetró en el vientre muscular sin dividirse.

Los RS penetraron a niveles variables en el vientre muscular y los promedios de las distancias respecto a la LBE están expresados en las Tablas II y III.

La localización de los puntos de penetración de los RS, tanto de la cabeza corta como la cabeza larga del músculo en cuestión y considerando las muestras que recibieron sólo un ramo principal, ingresaron a nivel del tercio medio de la distancia Acromion-LBE, tanto en el lado derecho como en el izquierdo. La ubicación dentro del tercio medio se muestran en las tablas IV, V,VI y VII, respectivamente.

Con respecto a los casos con dos ramos principales (R1 y R2) para la cabeza larga del músculo bíceps braquial del lado derecho, tanto el R1 como el R2 se dividieron en 2 RS en 4 casos; en otros 2 casos el R1 se dividió en 3 RS y en 5 RS en el otro, el R2 de uno de los casos mencionados se dividió en 3 RS y en el otro caso penetró directamente en el vientre muscular. En el lado izquierdo, el R1 de los 3 casos observados se dividió en 2 RS y el R2 de uno de ellos lo hizo en 2 RS, mientras que en los 2 casos restantes el R2 penetró directamente en su vientre muscular.

De los dos casos que tuvieron tres ramos principales (R1,R2,R3), en uno de ellos el R3 se dividió en 2 RS, los R1 y R2 penetraron directamente en el vientre muscular; en el caso restante, el R1 se dividió en 2 RS y el R2 y R3, penetraron directamente en el músculo.

Con respecto a la cabeza corta, lado derecho, en 2 casos se presentó con 2 ramos principales, en uno de ellos el R1 se dividió en 3 RS y en el otro penetró directamente en el músculo; en el primero de ellos el R2 se dividió en 2 RS y en el segundo penetró directamente en el músculo; en un caso se presentaron 3 ramos principales, donde R1 y R2 penetraron directamente en el músculo y el R3 se dividió en 3 RS. En el lado izquierdo, en 3 casos se presentaron 2 ramos principales, donde el R1 de dos de ellos se dividió en 2 RS y el restante penetró directamente en el vientre muscular; un caso tuvo 3 ramos principales, donde R1 penetró directamente en el músculo, R2 se dividió en 2 RS y R3 en 3 RS. 


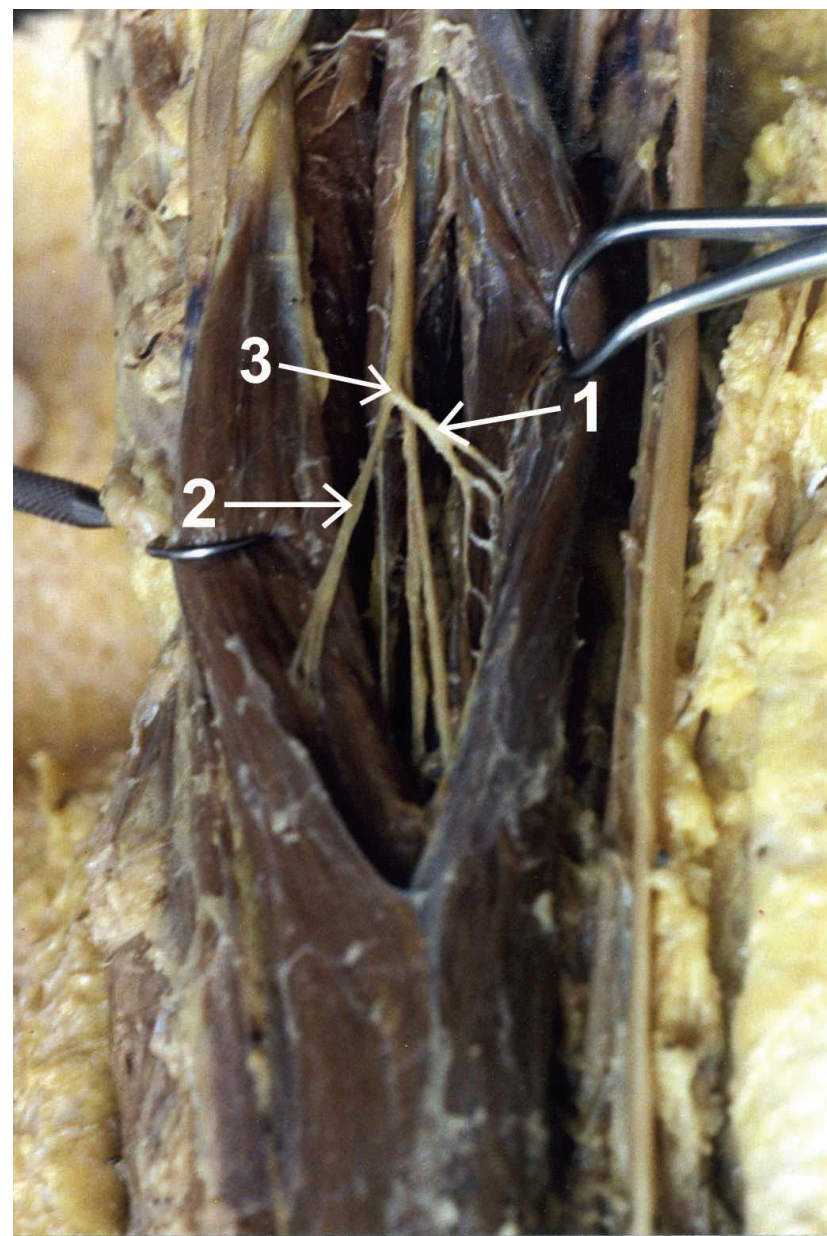

Fig. 1. Nervios de la cabeza corta (1) y de la cabeza larga (2) del músculo bíceps braquial, originándose de un tronco común (3) procedente del nervio musculocutáneo.

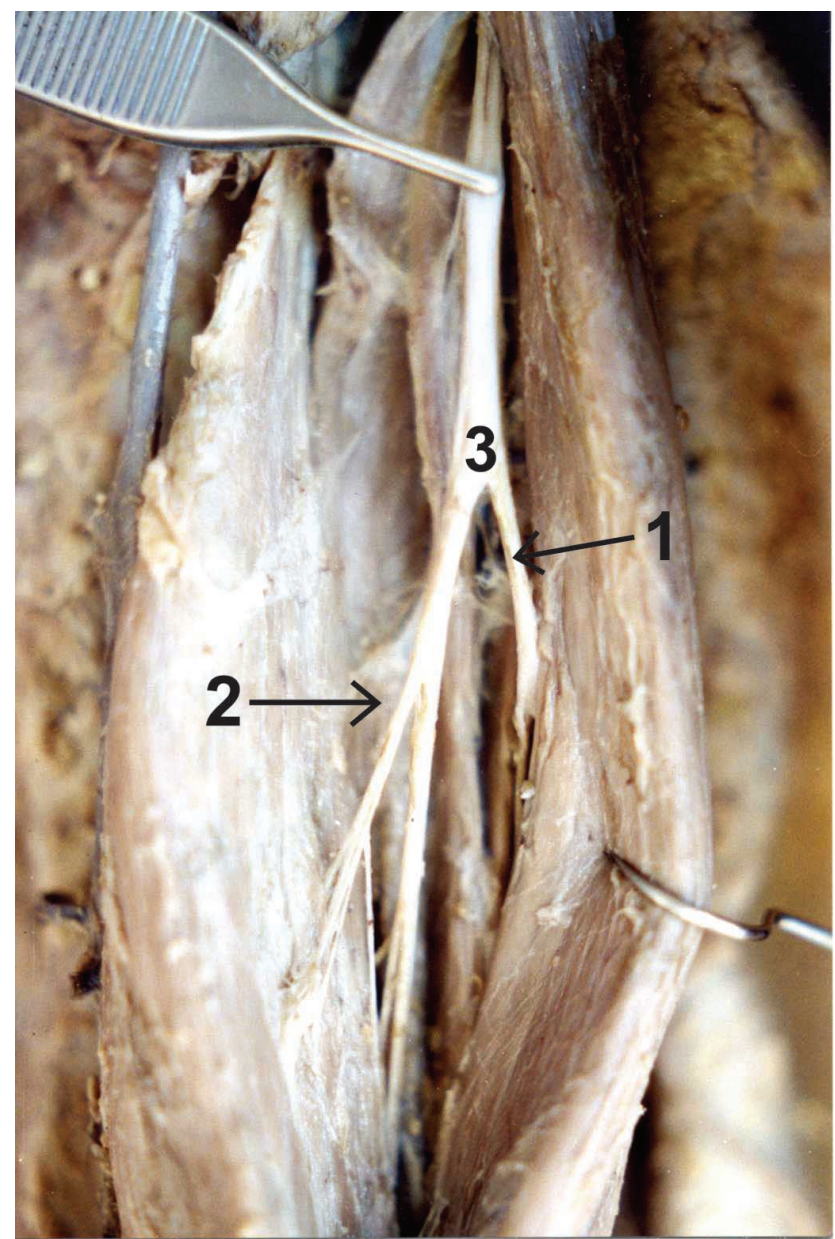

Fig. 2. Nervios de la cabeza corta (1) y de la cabeza larga (2) del músculo bíceps braquial, originándose en forma independiente desde el nervio musculocutáneo (3).

Tabla I. Promedios de las distancias entre el origen del ramo muscular y la LBE (en mm)

\begin{tabular}{lcccc}
\hline \multirow{2}{*}{ M. Bíceps braquial } & \multicolumn{2}{c}{ Lado derecho } & \multicolumn{2}{c}{ Lado izquierdo } \\
& Promedio & DS & Promedio & DS \\
\hline Cabeza larga & 162,0 & 12,4 & 161,4 & 10,9 \\
Cabeza corta & 160,1 & 11,9 & 158,5 & 13,6 \\
\hline
\end{tabular}

* Al comparar los lados derecho e izquierdo, no se observaron diferencias estadísticamente significativas.

Tabla II. Promedios de las distancias entre puntos de penetración de los RS y la LBE en la cabeza larga del m. bíceps braquial (en mm).

\begin{tabular}{ccccccc}
\hline Ramos & \multicolumn{3}{c}{ Lado derecho } & \multicolumn{3}{c}{ Lado izquierdo } \\
& Promedio & DS & N $^{\text {o }}$ casos & Promedio & DS & N $^{\text {o }}$ casos \\
\hline RS1 & 134,7 & 15,7 & 15 & 139,6 & 10,9 & 19 \\
RS2 & 125,9 & 18,9 & 13 & 128,3 & 12,4 & 20 \\
RS3 & 119,1 & 13,3 & 9 & 120,0 & 7,4 & 10 \\
RS4 & 123,4 & 13,3 & 4 & - & - & - \\
RS5 & 138,7 & - & 2 & - & - & - \\
\hline
\end{tabular}

* Al comparar los lados derecho e izquierdo, no se observaron diferencias estadísticamente significativas. 


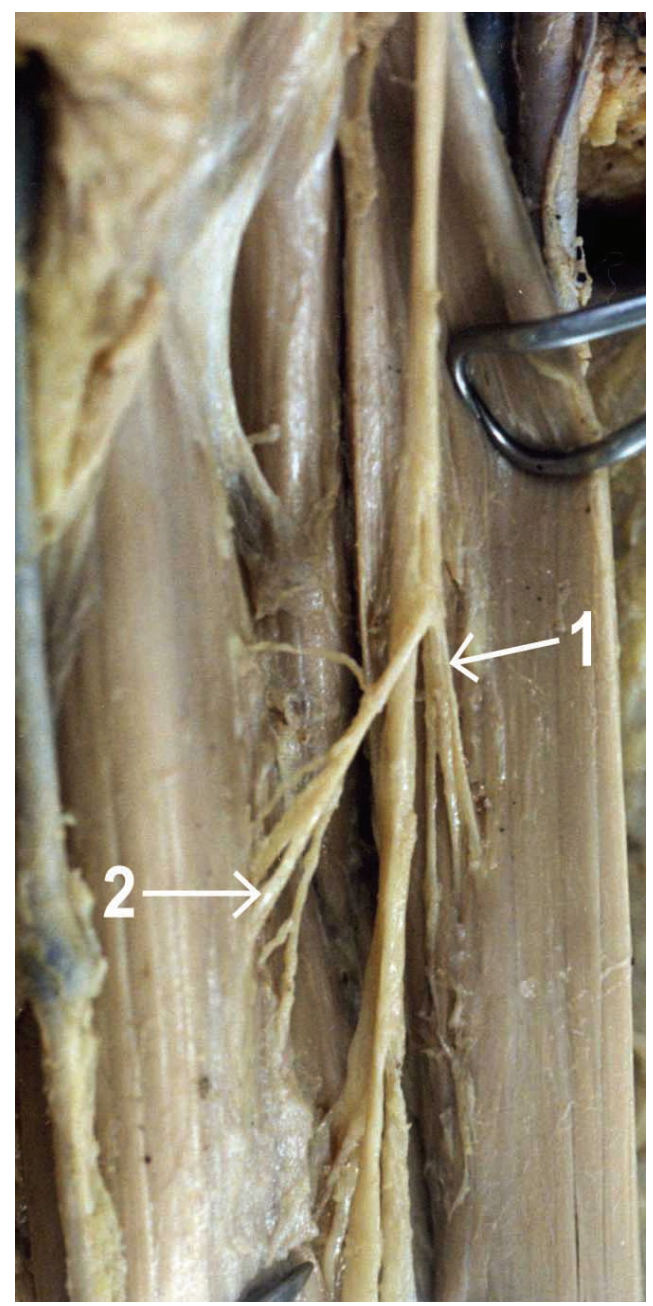

Fig. 3. Ramos secundarios en la cabeza corta (1) y en la cabeza larga (2) del músculo bíceps braquial.

\section{DISCUSIÓN}

Los estudios sobre la inervación de los músculos esqueléticos son de gran importancia en las intervenciones quirúrgicas y sobretodo cuando algún músculo ha sido afectado por parálisis y requiere para su recuperación de tratamientos de electroestimulación. Además, son necesarios cuando se requiere inyectar sustancias que impidan la contracción espástica derivada de lesiones en la parte alta del SNC. Sin embargo, para efectuar tales procedimientos se requiere de un conocimiento detallado de los puntos de ingreso de los ramos nerviosos en el vientre muscular, asociado a estructuras fácilmente localizables en la superficie corporal, principalmente, partes de huesos. Este punto de ingreso conocido como punto motor, donde su estimulación permite realizar la máxima contracción muscular efectiva, no siempre corresponde al ramo que es descrito como principal, ya que éste, generalmente se divide en ramos menores o secundarios que ingresan en diversos puntos del vientre muscular, ocupando una amplia área en el mismo (Olave et al., 2002; Olave \& Braga, 2003; Olave, 2004). Así, podemos encontrar que un músculo puede tener uno, dos o más puntos motores. Por otra parte, algunos músculos pueden recibir más de un ramo principal, ingresando a diversos niveles en su vientre.

La literatura nos indica que es posible que el músculo bíceps braquial esté formado por más de dos cabezas (Asvat et al., 1993; Vásquez et al., 2003; Abu-Hijleh, 2005), lo que lógicamente cambia el número de ramos destinados a su inervación. Esta que depende generalmente del nervio musculocutáneo y que a veces el referido nervio puede

Tabla III. Promedios de las distancias entre puntos de penetración de los RS y la LBE en la cabeza corta del m. bíceps braquial (en mm).

\begin{tabular}{ccccccc}
\hline Ramos & \multicolumn{3}{c}{ Lado derecho } & \multicolumn{3}{c}{ Lado izquierdo } \\
& Promedio & DS & N $^{\text {c } \text { casos }}$ & Promedio & DS & $N^{\text {o casos }}$ \\
\hline RS1 & 148,0 & 12,1 & 20 & 147,8 & 15,4 & 18 \\
RS2 & 140,0 & 11,4 & 19 & 134,0 & 12,0 & 18 \\
RS3 & 130,0 & 16,4 & 10 & 131,4 & 12,4 & 7 \\
\hline
\end{tabular}

* Al comparar los lados derecho e izquierdo, no se observaron diferencias estadísticamente significativas.

Tabla IV. Localización de los puntos motores de la cabeza corta del músculo bíceps braquial en las zonas del tercio medio derecho.

\begin{tabular}{|c|c|c|c|c|c|c|c|c|c|}
\hline \multirow{3}{*}{$\begin{array}{c}\text { ramos } \\
\text { secundarios }\end{array}$} & \multicolumn{3}{|c|}{ PP } & \multicolumn{3}{|c|}{ P M } & \multicolumn{3}{|c|}{ PD } \\
\hline & \multicolumn{3}{|c|}{$\mathrm{N}^{\mathrm{o}}$ de casos } & \multicolumn{3}{|c|}{$\mathrm{N}^{\circ}$ de casos } & \multicolumn{3}{|c|}{$\mathrm{N}^{\mathrm{o}}$ de casos } \\
\hline & $\mathrm{Zp}$ & $\mathrm{Zm}$ & $\mathrm{Zd}$ & $\mathrm{Zp}$ & $\mathrm{Zm}$ & $\mathrm{Zd}$ & $\mathrm{Zp}$ & $\mathrm{Zm}$ & $\mathrm{Zd}$ \\
\hline RS1 & & & 3 & 6 & 8 & 3 & & & \\
\hline $\mathrm{RS} 2$ & & & & 3 & 8 & 7 & & & \\
\hline RS3 & & & & 1 & 3 & 2 & 3 & 1 & \\
\hline RS4 & & & & & 1 & 1 & & & \\
\hline
\end{tabular}


OLAVE, E.; GABRIELLI, C. \& BRAGA, M. T. T. Padrones de inervación y localización biométrica de los puntos motores del músculo bíceps braquial en el hombre. Int. J. Morphol., 27(2):495-501, 2009.

Tabla V. Localización de los puntos motores de la cabeza corta del músculo bíceps braquial en las zonas del tercio medio izquierdo.

\begin{tabular}{|c|c|c|c|c|c|c|c|c|c|}
\hline \multirow{2}{*}{$\begin{array}{c}\text { ramos } \\
\text { secundarios }\end{array}$} & \multicolumn{3}{|c|}{$\begin{array}{c}\mathrm{PP} \\
\mathrm{N}^{\circ} \text { de casos }\end{array}$} & \multicolumn{3}{|c|}{$\begin{array}{c}\text { P M } \\
\mathrm{N}^{\mathrm{o}} \text { de casos }\end{array}$} & \multicolumn{3}{|c|}{$\begin{array}{c}\text { PD } \\
\mathrm{N}^{\circ} \text { de casos }\end{array}$} \\
\hline & $\mathrm{Zp}$ & $\mathrm{Zm}$ & $\mathrm{Zd}$ & $\mathrm{Zp}$ & $\mathrm{Zm}$ & $\mathrm{Zd}$ & $\mathrm{Zp}$ & $\mathrm{Zm}$ & $\mathrm{Zd}$ \\
\hline RS1 & & 1 & 3 & 5 & 7 & 1 & 2 & & \\
\hline $\mathrm{RS} 2$ & & & & 2 & 5 & 8 & & & 1 \\
\hline RS3 & & & & & 3 & 2 & & 1 & \\
\hline RS4 & & & & & & 1 & & & 1 \\
\hline
\end{tabular}

$\mathrm{PP}=$ parte proximal $; \mathrm{PM}=$ parte media $; \mathrm{PD}=$ parte distal $\mathrm{Zp}=$ zona proximal $; \mathrm{Zm}=$ zona media $; \mathrm{Zd}=$ zona distal

Tabla VI. Localización de los puntos motores de la cabeza larga del músculo bíceps braquial en las zonas del tercio medio derecho.

\begin{tabular}{|c|c|c|c|c|c|c|c|c|c|}
\hline \multirow{2}{*}{$\begin{array}{c}\text { ramos } \\
\text { secundarios }\end{array}$} & \multicolumn{3}{|c|}{$\begin{array}{c}\mathrm{PP} \\
\mathrm{N}^{\mathrm{o}} \text { de casos }\end{array}$} & \multicolumn{3}{|c|}{$\begin{array}{c}\mathrm{P} \mathrm{M} \\
\mathrm{N}^{\circ} \text { de casos }\end{array}$} & \multicolumn{3}{|c|}{$\begin{array}{c}\mathrm{PD} \\
\mathrm{N}^{\mathrm{o}} \text { de casos }\end{array}$} \\
\hline & $\mathrm{Zp}$ & $\mathrm{Zm}$ & $\mathrm{Zd}$ & $\mathrm{Zp}$ & $\mathrm{Zm}$ & $\mathrm{Zd}$ & $\mathrm{Zp}$ & $\mathrm{Zm}$ & $\mathrm{Zd}$ \\
\hline $\mathrm{RS} 1$ & & & 1 & 1 & 5 & 2 & 4 & 2 & \\
\hline $\mathrm{RS} 2$ & & & & & 3 & 2 & 5 & 1 & 2 \\
\hline RS3 & & & & & & 4 & & 4 & \\
\hline RS4 & & & & & & 2 & 2 & & \\
\hline RS5 & & & & & & 1 & & & \\
\hline RS6 & & & & & & & 1 & & \\
\hline
\end{tabular}

Tabla VII. Localización de los puntos motores de la cabeza larga del músculo bíceps braquial en las zonas del tercio medio izquierdo.

\begin{tabular}{|c|c|c|c|c|c|c|c|c|c|}
\hline \multirow{2}{*}{$\begin{array}{c}\text { ramos } \\
\text { secundarios }\end{array}$} & \multicolumn{3}{|c|}{$\begin{array}{c}\mathrm{PP} \\
\mathrm{N}^{\circ} \text { de casos }\end{array}$} & \multicolumn{3}{|c|}{$\begin{array}{c}\text { P M } \\
\mathrm{N}^{\mathrm{o}} \text { de casos }\end{array}$} & \multicolumn{3}{|c|}{$\begin{array}{c}\mathrm{PD} \\
\mathrm{N}^{\mathrm{o}} \text { de casos }\end{array}$} \\
\hline & $\mathrm{Zp}$ & $\mathrm{Zm}$ & $\mathrm{Zd}$ & $\mathrm{Zp}$ & $\mathrm{Zm}$ & $\mathrm{Zd}$ & $\mathrm{Zp}$ & $\mathrm{Zm}$ & $\mathrm{Zd}$ \\
\hline RS1 & & & & 4 & 6 & 9 & 1 & & \\
\hline $\mathrm{RS} 2$ & & & & & 5 & 3 & 11 & & 1 \\
\hline RS3 & & & & & & 1 & 6 & 2 & 1 \\
\hline RS4 & & & & & & & & 1 & \\
\hline RS5 & & & & & & & & & 1 \\
\hline
\end{tabular}

$\mathrm{PP}=$ parte proximal $\mathrm{PM}=$ parte media $\mathrm{PD}=$ parte distal $\mathrm{Zp}=$ zona proximal $\mathrm{Zm}=$ zona media $; \mathrm{Zd}=$ zona distal.

no estar presente y en tal caso, el músculo recibe ramos directos del plexo braquial o del nervio mediano (Nakatani \& Tanaka, 1997; Gümüsburun \& Adigüzel, 2000; Rao \& Chaudhary, 2001) En esta investigación no se observó más que dos cabezas en su constitución y los ramos de inervación procedieron del nervio musculocutáneo.

Con respecto al número de ramos que recibe cada una de las cabezas constituyentes del músculo bíceps braquial se constató que en la mayor parte de los casos, 35 para la cabeza larga y 39 para la corta, recibieron 1 ramo principal, pudiendo originarse a partir de un tronco común o como ramo independiente desde el nervio musculocutáneo, disposición comentada por Tountas \& Bergman (1993) los cuales indicaron que el nervio musculocutáneo envia un ramo a cada cabeza, aunque los ramos pueden ser encontrados jun- tos por una variable distancia como tronco común. Sin embargo, este ramo dirigido a cada cabeza del músculo en cuestión, se dividió previamente a ingresar en el vientre, con frecuencia, en 2 o 3 ramos menores o secundarios, disposición concordante con lo expresado por Sunderland (1985).

Diversos autores han estudiado la inervación del músculo en cuestión, determinando el origen de los nervios para las cabezas que le componen, entre ellos Yang et al. que clasificaron esta distribución en 3 tipos, donde el tipo I corresponde a un tronco común que emite un nervio para cada cabeza y que encontró en 20 de 24 muestras $(83,3 \%)$, porcentaje alto en relación al hallazgo de Kwolczak-McGrath et al. que estudiaron 40 fetos y cuyo porcentaje fue de $47,5 \%$, semejante al 43,1\% encontrado en la presente serie. Pacha Vicente et al. describió este tipo en $60,5 \%$. 
El tipo II de Yang et al. correspondía a ramos independientes originándose del ramo principal del nervio musculocutáneo, disposición que sólo encontraron en 2 muestras $(8,3 \%)$, que Pacha Vicente et al. observaron en $27,9 \%$ y Kwolczak-McGrath et al. en 42,5\%, porcentajes menores a este estudio el cual arrojó un $58,7 \%$, pero que permiten determinar que la inervación de los componentes del músculo en estudio puede provenir tanto de un ramo común como de ramos independientes derivados del nervio musculocutáneo.

El tipo III de Yang et al., en donde un ramo se desprende de la parte distal del nervio musculocutáneo para el vientre común del bíceps y que los autores citados previamente encontraron en alrededor de $10 \%$, no fue observado en nuestras muestras

Con respecto a la posición de los puntos motores, el conocer la distancia entre el acromion y la línea biepicondilar ha permitido dividir a ésta en tres partes y cada una de ellas nuevamente en tres, pudiendo con ello identificar la zona en que podemos encontrar los puntos de ingreso en el vientre muscular.

La localización de los puntos de penetración de los
RS, tanto de la cabeza corta como la cabeza larga del músculo en cuestión y considerando las muestras que recibieron sólo un ramo principal, ingresaron a nivel del tercio medio de la distancia Acromion-LBE, tanto en el lado derecho como en el izquierdo. Es interesante observar que en la cabeza corta los puntos motores se localizaron en mayor número de muestras a nivel de la parte media del tercio medio de la distancia acromion-LBE, tanto en el lado derecho como en el izquierdo, mientras que en la cabeza larga esta concentración se fue distribuyendo más hacia la parte distal. Información general sobre el punto de penetración del ramo en el músculo estudiado es mostrada en el texto de Sunderland, donde se indica que en la mitad de los casos penetra a nivel de la mitad del brazo y aproximadamente en un tercio, lo hace distal a ésta, sin especificar distancias respecto a un punto de referencia.

Estos datos permiten calcular aproximadamente el lugar donde aplicar una punción para el tratamiento con neurolíticos o anestésicos en pacientes con lesiones que afecten la función del músculo, además de aportar el conocimiento necesario para efectuar estudios con electromiografía. La consideración de los presentes resultados pueden llevar a un mejor manejo en esos procedimientos.

OLAVE, E.; GABRIELLI, C. \& BRAGA, M. T. T. Innervation patterns and biometric localization of the motor points of the brachial biceps muscle in man. Int. J. Morphol., 27(2):495-501, 2009.

SUMMARY: The biceps brachii muscle innervation is important due to its constitution and its function in the elbow joint, mainly when there are in spinal nerves damages or high spinal cord. The purpose of this study was determine with details the nerves disposition of this muscle and locate its motor points. The upper limbs of 23 formolized cadavers of adult Brazilian individuals were studied, in whose it recorded the number of branches, length, origin levels and motor point localization. The localization were recorded using as reference point a line between the humerus epicondyles. The musculocutaneous nerve send a branch for a studied muscle as a common trunk that divides in two branches, each to a different head of the biceps in 19 cases $(41.3 \%)$; independent branches for each head were observed in the other cases (58.7\%). The long head received one branch in 35 cases $(76.1 \%)$; two branches (B1,B2) in 9 cases (19.6\%) and three branches (B1,B2,B3) in 2 cases $(4.3 \%)$. Each branch divided in secondary branches (SB). In the short head the principal branch divided in two SB in 20 cases; in three SB in 13 cases; in fourth SB in 4 cases and not dividing in 2 cases. In the long head, the principal branch divided in two SB in 16 cases; in three SB in 13 cases; in fourth SB in 3 cases; in five SB in one case and in sixth SB in one case and not dividing in one case. The SB pierce in the muscular belly to different levels. These results can be to use as reference for the puncture procedures in the motor points or in electromyography studies.

KEY WORDS: Brachial biceps muscle; Biometry; Motor points.

\section{REFERENCIAS BIBLIOGRÁFICAS}

Abu-Hijleh, M. Three-headed biceps brachii muscle associated with duplicated musculocutaneous nerve. Clin. Anat., 18:376-9, 2005.

Asvat, R.; Candler, P. \& Sarmiento, E. High incidence of the third head of biceps brachii in South African populations. J. Anat., 182:101-4, 1993

Buchanan T. \& Ericsson J.C. Selective block of the brachialis motor points. An anatomic investigations of musculocutaneous nerve branching. Reg Anesth., 21(2):89-92, 1996. 
Chiarapattanakon,P; Leechavengvongs, S.; Witoonchart, K.; Uerpairojkit, C.; Thuvasethakul, P. Anatomy and internal topography of the musculocutaneous nerve: the nerves to the biceps and brachialis muscles. J. Hand Surg. (Am), 23(2):250-5, 1998

Gümüsburun, E. \& Adigüzel, E. A variation of the brachial plexus characterized by the absence of the musculocutaneous nerve: a case report. Surg. Radiol. Anat., 22(1):63-5, 2000.

Kwolczak-Mc Grath, A.; Kolesnik, A. \& Ciszek, B. Anatomy of branches of musculocutaneous nerve to the biceps and brachialis in human fetuses. Clin. Anat., 21(2):142-6, 2008.

Moore, Kl. \& Dalley, AF. Anatomía con orientación Clínica. 4a Ed. Buenos Aires, Panamericana, 2002.

Nakatani, T. \& Tanaka, S. Absence of the musculocutaneous nerve with innervation of coracobrachialis, bíceps brachii, brachialis and the lateral border of the forearm by branches from the lateral cord of the bracial plexus. $J$. Anat., 191:459-60, 1997

Olave , E.; Gabrielli, C.; Braga, MTT \& del Sol, M. Aspectos biométricos de los ramos motores del nervio musculocutáneo para el músculo braquial. Rev. Chil Anat, 20(2): 231-236, 2002.

Olave, E. \& Braga, MTT. Inervación del músculo semitendinoso, biometría de sus ramos y localización de sus puntos motores. Int. J. Morphol., 21(2):161-5, 2003.

Olave, E. Aspectos anatómicos y biométricos de la inervación del músculo coracobraquial y sus puntos motores. Int. J. Morphol., 22(4):323-6, 2004.

Pacha Vicente, D.; Forcada Calvet, P.; Carrera Burgaya, A.; Llusá Pérez, M. Innervation of bíceps brachii and brachialis: Anatomical and surgical approach. Clin. Anat., 18(3):186-194, 2005.

Rao, P. V. \& Chaudhary, S.C. Absence of musculocutaneous nerve: two case reports. Clin. Anat., 14:31-5, 2001.

Sunderland, S. Nervios periféricos y sus lesiones. Barcelona, Salvat, 1985.

Testut, L. \& Latarjet, A. Tratado de Anatomía Humana. Barcelona, Salvat, 1969.

Tountas, Ch. \& Bergman, R. Anatomic variations of the upper extremity. New Cork, Churchill Livingstone, 1993.
Vásquez, T.; Rodríguez-Niedenführ, M.; Parkin, I. \& Sañudo, J.R. A rare case of a four-headed biceps brachii muscle with a double piercing by the musculocutaneous nerve. Surg. Radiol. Anat., 25:462-4, 2003.

Williams, PL; Warwick, R.; Dyson, M. \& Bannister, LH. Gray Anatomia. $37^{\text {th }}$ Ed. Rio de Janeiro, Guanabara Koogan, 1995.

Yang, Z.; Pho, R.; Kour, A. \& Pereira, B. The musculocutaneous nerve and its branches to the bíceps and brachialis muscle. J. Hand Surg., 20A:671-675, 1995.

Dirección para correspondencia:

Prof. Dr. Enrique Olave R.

Facultad de Medicina

Universidad de La Frontera

Casilla 54-D

Temuco - CHILE

Email: eolave@ufro.cl

Recibido : 19-01-2009

Aceptado: 03-03-2009 
